\title{
Palmier à huile : défis et questions à la recherche
}

\author{
Alain RIVAL \\ Cirad, \\ UMR DIADE, \\ 911 Avenue Agropolis, \\ F-34394 Montpellier Cedex5, \\ France \\ <alain.rival@cirad.fr>
}

Article reçu le 15 février 2013

Accepté le 10 mars 2013

\section{Une filière globale, ancrée au Sud}

Le palmier à huile, Elaeis guinneensis Jacq., est une plante monocotylédone pérenne; ce n'est donc pas un arbre, mais une herbe géante. Il est exclusivement cultivé dans les zones tropicales humides où il constitue une importante source de devises pour les économies locales, à la fois pour l'exportation et en tant que matière première pour l'industrie locale.

La production mondiale d'huile de palme est aujourd'hui majoritairement assurée par deux pays, I'Indonésie et la Malaisie, qui totalisent à eux seuls $87 \%$ des approvisionnements (figure 1).

La consommation est tirée par les pays du Sud, à la fois portée par la croissance démographique et l'élévation du niveau de vie dans les pays émergents à forte population comme I'Inde, I'Indonésie et la Chine. La consommation européenne pèse pour $12 \%$ dans la balance mondiale, les USA pour $3 \%$ (figure 2).

\section{Une expansion avérée et prévisible}

La consommation globale en corps gras per capita a plus que doublé entre 1975 et 2010, passant de $11 \mathrm{~kg}$ en 1976 à

\begin{abstract}
The oil palm: challenges and questions to research
Since several decades, the oil palm has been able to transform tropical landscapes and livelihoods. The oil palm is an outstanding oil crop producing very high oil yields and showing an amazing agronomic plasticity: these are valuable assets in a context of food safety and ecological intensification. The palm oil production now constitutes a global commodity chain which is at the focus point of North/South challenges such as certification, traceability or environmental and sanitary impacts. Palm oil is playing an increasing part in the production of edible oils; such an expansion must now comply with sustainability criteria backed by robust and shared scientific results. The sustainable development of oil palm cultivation thus generates many questions to research, not only in the traditional domains of plant science, but also in the fields of socio-economy and social and human sciences.
\end{abstract}

Key words: palm oil, RSPO, sustainability

24,7 kg par habitant et par an en 2009. Corley (2009) estime que la production en corps gras devra doubler d'ici 2050 (figure 3), malgré les incertitudes liées à l'évolution de la consommation per capita et sur le rôle joué par les biocarburants, intimement lié à l'évolution du cours des énergies fossiles.

Les cours mondiaux sont à la hausse depuis plus d'une décennie (figure 4) expliquant un engouement sans précédent chez les planteurs du Sud et les agro-industriels. En outre, on note depuis 2008 une évolution parallèle du cours des huiles végétales candidates potentielles au biodiesel et de celui du pétrole brut (Jacquemard, 2012).

\section{Des utilisations multiples}

L'huile de palme peut se substituer à la plupart des autres huiles végétales et possède de très nombreux usages :

- $80 \%$ pour l'agroalimentaire : huiles de table, huiles de friture, margarines, matières grasses pour boulangerie, pâtisserie, et tout type de préparation alimentaire...;

- 19 \% pour l'oléochimie : cosmétiques, savonneries, lubrifiants et graisses, bougies, produits pharmaceutiques, cuir, surfactants, agrochimie, peintures et laques, électronique, etc.

$-1 \%$ pour le biodiesel.

\section{Une oléagineuse d'exception}

Le palmier à huile offre des rendements en huile à I'hectare exceptionnels : ils atteignent 3,5 tonnes/hectare en moyenne mondiale, près de $7 \mathrm{~T} / \mathrm{ha}$ dans les meilleures plantations en Asie du SudEst, et plus de $10 \mathrm{~T} / \mathrm{ha}$ dans les meilleurs essais génétiques en cours au Cirad. Ainsi, en occupant seulement $7 \%$ des surfaces agricoles en oléagineux, le palmier à huile assure $39 \%$ de la production mondiale en huile végétale, une fraction bien plus faible que celle consacrée au soja (61\%), au colza (18\%) ou au tournesol (14\%). L'huile de palme présente les coûts de production les moins élevés des huiles végétales, inférieurs de $20 \%$ à ceux du soja.

\section{Une cohabitation délicate}

La biologie du palmier à huile, originaire du Golfe de Guinée, impose une

Pour citer cet article : Rival A. Palmier à huile : défis et questions à la recherche. OCL $2013 ; 20(3)$ : 133-142. doi : 10.1684/ocl.2013.0506 


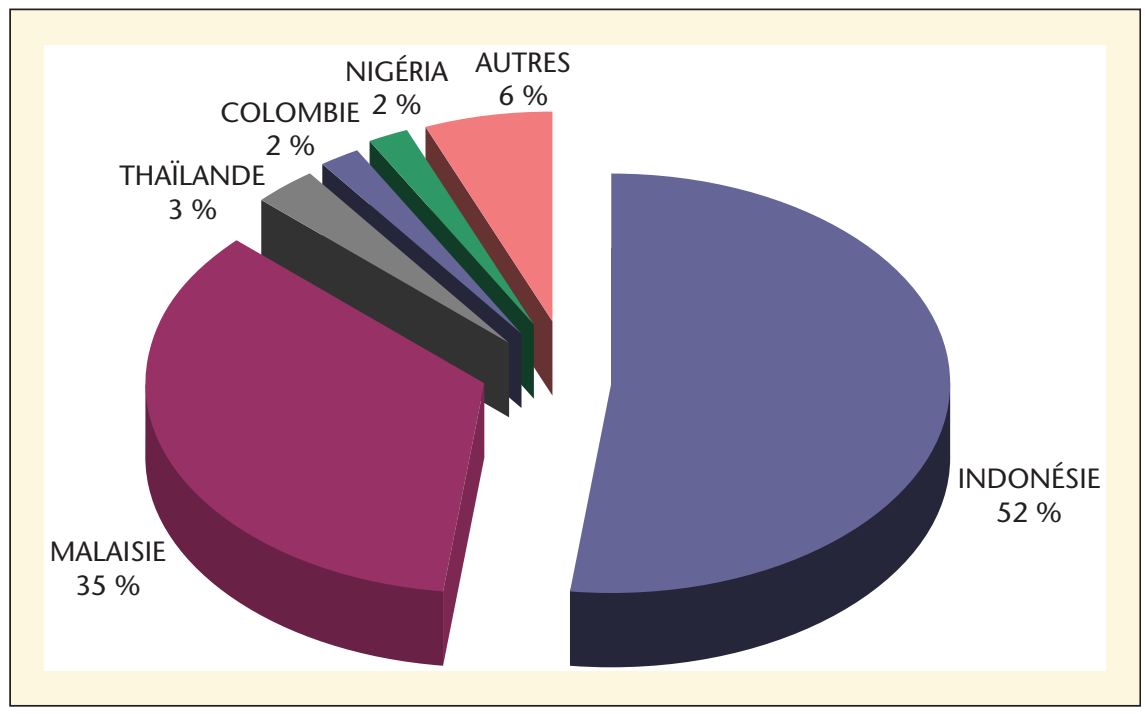

Figure 1. Répartition de la production mondiale d'huile de palme.

distribution strictement intertropicale de son exploitation, donc une cohabitation forcée avec des zones de forte biodiversité, dont le Bassin du Congo, le Bassin amazonien ou l'île de Bornéo (figure 5).

Cette cohabitation délicate n'est pas le propre du palmier à huile. Elle affecte également les autres oléagineuses de grande culture. Ainsi la culture du soja (OGM à $75 \%$ ) doit aussi faire face à d'importantes contraintes environnementales, notamment au Brésil où les surfaces en production sont passées de 1,7 millions à 21,7 millions d'hectares en moins de 40 ans.

La relation entre plantations de palmier et déforestation n'est pas directe, ni automatique. Les concessions sont accordées par les pouvoirs publics aux compagnies forestières, qui exploitent laissées en friche et régénèrent des forêts secondaires, ou peuvent évoluer en savane ou en terres cultivées sous l'action de l'homme. le bois. Les forêts dégradées sont alors

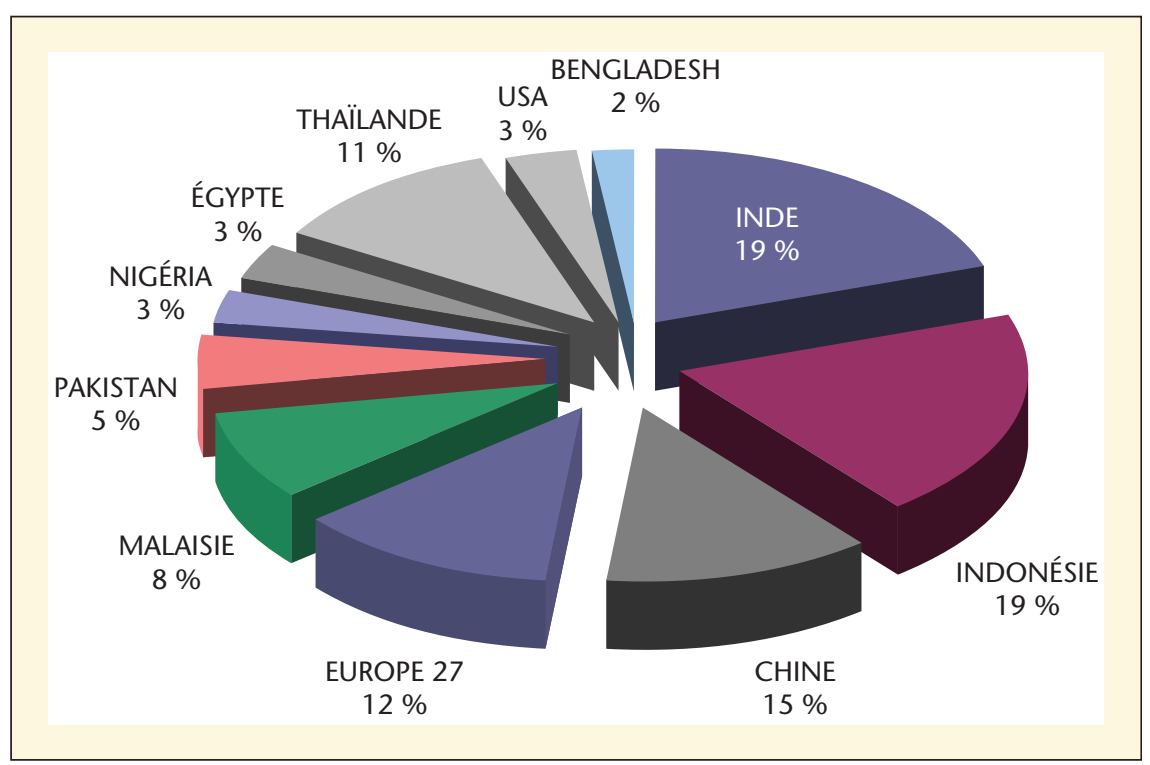

Figure 2. Répartition de la consommation mondiale d'huile de palme.
Les espaces déforestés ne sont que partiellement reconvertis en plantations de palmier. Ainsi, sur les 21 millions $d$ 'hectares de forêt primaire qui ont disparu en Indonésie entre 1990 et 2005, seulement 3 millions correspondent à la création de palmeraies. Cette surface est très importante, mais on ne peut attribuer la totalité de la déforestation à la mise en place de palmeraies. Toutefois, cette relation directe a tendance à s'aggraver dans de nouveaux fronts pionniers, comme à Bornéo où près de $30 \%$ des forêts primaires abattues ont été converties en palmier à huile (Carlson et al., 2012), alors que les nouvelles plantations sont responsables en moyenne des $10 \%$ de la déforestation enregistrée en Indonésie et Malaisie (FAO, 2010). Les extensions de plantations doivent donc se programmer avec une extrême vigilance.

Il est nécessaire en premier lieu d'identifier des forêts à haute valeur de conservation et de les entourer de zones tampons incluant des agroforêts permettant une activité humaine contrôlée : chasse, cueillette, cultures vivrières, fruitiers, caoutchouc, plantes médicinales ou même écotourisme. Cette pratique permet d'éviter une réduction drastique de la biodiversité consécutive à l'ouverture d'espaces agricoles en limite directe de forêt primaire. La culture du palmier à huile est alors intégrée à une planification du paysage en concertation avec les populations locales (Koh et al., 2009).

L'enjeu majeur, comme le souligne le WWF (2011), est bien de passer de la déforestation à la nécessaire durabilité.

\section{L'Afrique : un nouveau front}

L'Indonésie planifie de doubler sa production d'ici 2020 et l'Afrique (Golfe de Guinée et bassin du Congo) se présente comme la nouvelle frontière (650 000 ha en projet au Liberia).

Les grands programmes de plantation en cours d'élaboration en Afrique (Liberia, Angola, Gabon, Cameroun) font peser une menace croissance sur les forêts (WWF, 2011 ; Sayer et al., 2012). En effet, le nombre croissant de régulations prévenant la conversion des forêts naturelles, le manque de terres, la surveillance des grandes acquisitions foncières, et les espoirs suscités 


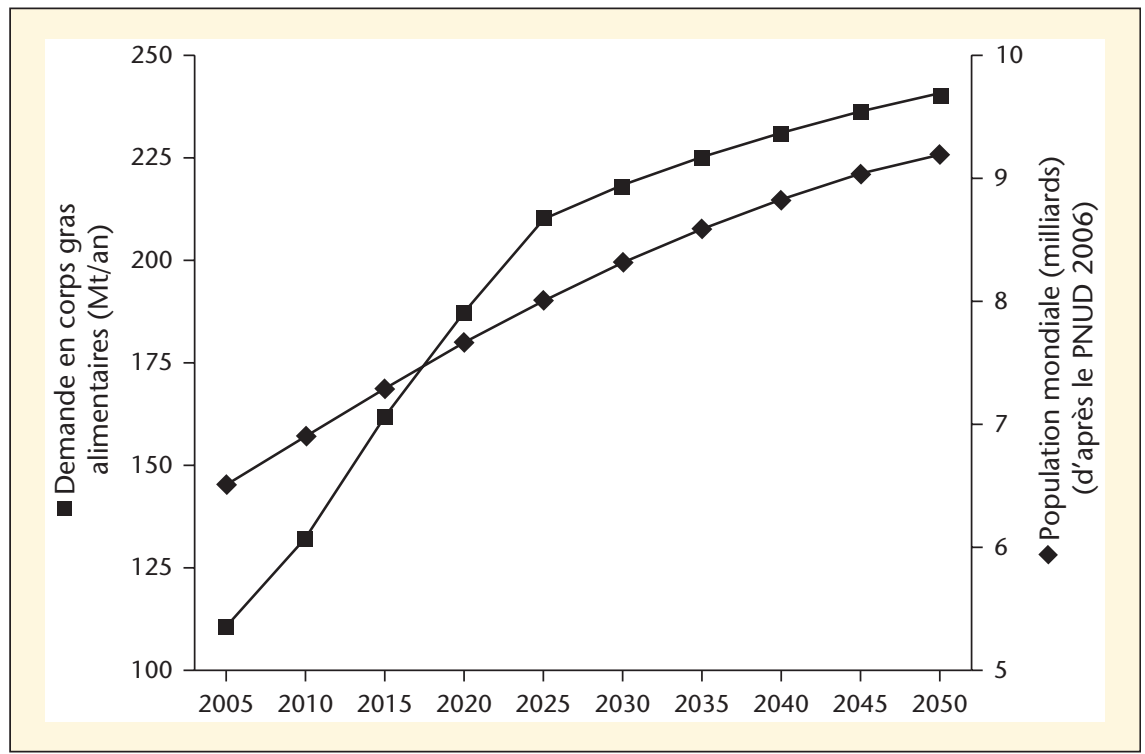

Figure 3. Évolution comparée de la population mondiale et de la demande en corps gras alimentaires (2005-2050).

par la Réduction des Emissions dues à la Déforestation et la Dégradation (REDD) dans les grands pays producteurs tels la Malaisie et l'Indonésie incitent les grandes compagnies asiatiques à diversifier leurs zones de production et à investir massivement en Afrique (Hoyle et Levang, 2012).

L'opportunité est unique de s'engager dans un développement concerté incluant, dès l'initiation des projets, les principaux acteurs socio-économiques : gouvernements, sociétés de plantations, bailleurs, organisations non gou- vernementales, centres de recherches, représentants des populations locales. Des outils de certification, sans doute imparfaits, existent et il importe qu'ils soient pris en considération en amont de l'attribution des concessions aux compagnies forestières et aux sociétés de plantation.

\section{L'intensification écologique}

Les périmètres déjà plantés en palmier sont souvent loin de présenter les

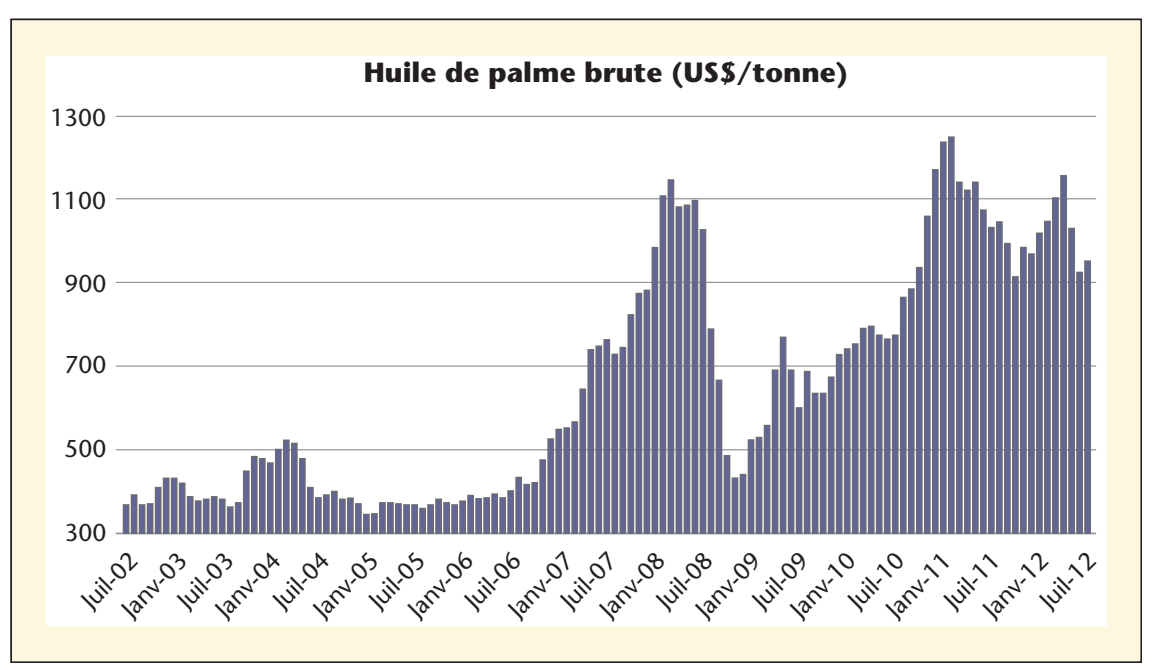

Figure 4. Évolution des cours mondiaux de l'huile de palme brute (2002-2012). rendements attendus (3,5 tonnes $\mathrm{d}$ 'huile/ha en moyenne mondiale, à comparer aux $10 \mathrm{~T} /$ ha produites par les meilleurs génotypes). Combler ce fossé entre rendement théorique et rendement réel est un des défis majeurs posés aux scientifiques et aux gestionnaires de plantation : il importe d'optimiser le fonctionnement des palmeraies installées, tout en impactant au minimum les hommes et l'environnement.

L'intensification écologique de la productivité requiert en premier lieu la mise à disposition de tous les planteurs familiaux comme industriels - de semences sélectionnées et certifiées, diffusant ainsi le progrès génétique issu de la recherche.

Ce progrès est estimé à un peu plus de $40 \%$ depuis 1960 , soit près de $1 \%$ par an (Durand-Gasselin et al., 2002). Le renouvellement du verger étant réalisé sur 30 ans, l'impact de nouvelles sélections sur la filière reste lent, même si l'intégration des biotechnologies a permis d'accélérer le transfert d'innovation, par l'adoption des techniques de clonage in vitro ou de sélection assistée par marqueurs ADN (Rival et Jaligot, 2010).

L'intensification écologique repose également sur la mise en place d'une fertilisation raisonnée, pour des raisons autant économique (60\% des coûts d'exploitation d'une palmeraie) que de respect des hommes et de l'environnement. Les fertilisants sont issus de la pétrochimie ou de sources minérales non renouvelables : ils constituent donc une ressource à l'avenir limité. L'enjeu est donc d'optimiser les apports d'engrais (minéraux ou organiques) afin qu'ils profitent au mieux à la plante au travers d'applications fractionnées et raisonnées, évitant ainsi que les surplus se retrouvent dans les nappes ou les eaux de surface. Ces besoins sont évalués au travers de prélèvements de sols et de feuilles dans les plantations. Leur analyse permet de donner des limites à I'utilisation d'engrais en fonction du climat, de la physiologie de la plante, de la nature du sol ou de l'âge de la plantation. Cette gestion raisonnée de la fertilisation n'est pas un concept nouveau, mais elle prend de l'ampleur depuis quelques années avec la prise de conscience que les hauts rendements doivent aller de pair avec la protection de l'environnement. 


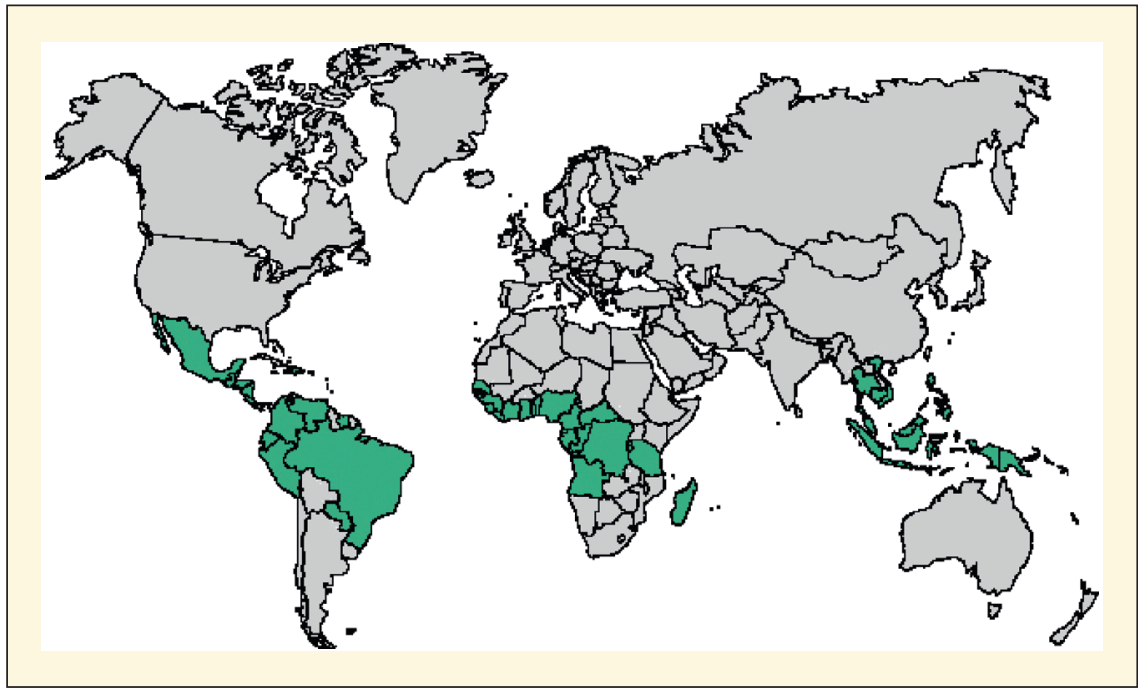

Figure 5. Répartition géographique de la culture du palmier à huile.

D'importants efforts sont effectués en ce qui concerne le compostage des déchets et le recyclage des effluents d'huilerie. Le lagunage des effluents liquides produit du méthane, un gaz à fort effet de serre qui est désormais exploité pour fournir du biogaz autour des usines d'extraction d'huile. Le considérable potentiel énergétique des sousproduits solides ( $12 \mathrm{~m}^{3}$ de méthane par tonne de fruits extraite, $15 \mathrm{~m}^{3}$ de méthane par $\mathrm{m}^{3}$ d'effluents liquides) est ainsi exploité et valorisé. Chaque $\mathrm{m}^{3}$ de biogaz généré permet de produire l'équivalent d'un demi-litre de carburant diesel. Cette pratique se généralise rapidement dans bon nombre de plantations et les installations neuves sont désormais équipées dès leur construction d'un dispositif de digestion des effluents et de capture de biogaz.

L'utilisation de produits phytosanitaires en élaeiculture se limite à des applications limitées d'herbicides dans les jeunes plantations, afin de limiter la croissance des plantes de couverture et d'établir les sentiers de récolte. Dans certaines régions, notamment en Asie du Sud-Est, I'emploi de raticides est courant, même s'il est de plus en plus substitué par l'introduction de prédateurs naturels comme les chouetteseffraies (Tyto alba). La part de produits phytosanitaires dans les coûts d'exploitation d'une palmeraie industrielle est inférieure à $1 \%$, contre 50 à $55 \%$ pour les dépenses en engrais et $30 \%$ pour les frais de récolte. En moyenne, les quantités totales épandues pour obtenir une tonne $d$ 'huile végétale sont de $10 \mathrm{~kg} / \mathrm{T}$ pour le soja contre $0,1 \mathrm{~kg} / \mathrm{T}$ pour le palmier à huile.

À ce jour, les endémies majeures qui frappent le palmier à huile ne sont pas traitées par des épandages massifs de produits phytosanitaires, les solutions biologiques ayant souvent été privilégiées. Ainsi, la fusariose en Afrique a été éradiquée grâce à des semences résistantes issues de programmes de sélection variétale classique. Les recherches vont dans le même sens concernant les attaques du champignon Ganoderma en Asie du Sud-Est et la lutte contre de la Pourriture du Cœur (un complexe de pathogènes) en Amérique latine, en parallèle de l'exploitation des ressources génétiques apportées par l'espèce de palmier amazonienne Elaeis oleifera.

Trois continents, trois maladies qui pour I'instant restent majoritairement confinées dans leur contexte d'origine, mais une situation qui reste menacée par la mondialisation des échanges de pollen, de semences et de plants.

L'intensification écologique de I'élaeiculture se heurte aux contraintes biologiques de la plante, qui la rendent difficilement mécanisable, donc exigeante en main d'œuvre (un homme pour 10 ha en moyenne). L'extraction de l'huile des fruits doit être immédiate, sous peine de perdre ses qualités physico-chimiques. Elle nécessite donc un réseau de collecte efficace et une organisation solide des bassins de récolte autour des usines d'extraction. Cette organisation se reflète dans les schémas d'exploitation associant un périmètre industriel et son usine (nucleus) à un nuage de petits exploitants regroupés autour de ce périmètre (plasma). Le schéma nucleus/plasma a été développé avec succès, notamment en Indonésie et en Papouasie NouvelleGuinée. II peut supporter des associations privé/public dans des actions de développement.

Les sciences humaines et sociales prennent une part croissante dans les recherches en cours. Elles répondent aux questions posées sur la résilience des systèmes d'exploitation et aux rapports de pouvoir dans la filière. La définition des variétés adaptées aux systèmes de culture et au type d'exploitants (familiaux ou industriels) ainsi que l'appropriation des innovations (matériel végétal ou bonnes pratiques agricoles) font l'objet de plusieurs programmes pluridisciplinaires en cours.

La filière est représentative de l'évolution des rapports Nord/Sud dans les filières agroalimentaires: les pays du Sud ne sont plus uniquement fournisseurs de matière première, ils offrent des marchés en pleine croissance, deviennent également transformateurs et normalisateurs (voir standards ISPO et MSPO).

\section{Impact sur la pauvreté rurale}

L'élaeiculture est capable de générer des revenus élevés et stables et de porter une classe moyenne rurale sur plusieurs générations, ce que peu de commodités tropicales sont capables de réaliser à ce jour.

Ainsi, à Sumatra (Indonésie), le revenu moyen à l'hectare sur un cycle complet d'une plantation s'élève à $2100 €$ pour le palmier à huile, contre seulement 200 $€ /$ ha pour une rizière. La comparaison des revenus du travail est encore plus frappante : $36 € /$ jour.homme pour le palmier à huile, et seulement $1,7 € /$ jour.homme pour le riz irrigué (Hoyle et Levang, 2012).

L'exploitation du palmier à huile repose sur des systèmes de culture très diversifiés, allant de l'exploitation familiale de quelques hectares au périmètre agro-industriel de plusieurs dizaines de 
milliers d'hectares. Plus de la moitié de I'huile de palme produite aujourd'hui provient de petits planteurs. Globalement, environ 3 millions de petits agriculteurs chefs de famille sont impliqués dans le secteur. En outre, on estime à 25 millions le nombre $\mathrm{d}^{\prime}$ Indonésiens vivant indirectement aujourd'hui de l'exploitation du palmier à huile (WWF, 2011).

Les petits exploitants (indépendants ou associés à des complexes agro-industriels) ont commencé à cultiver le palmier à huile en Indonésie en 1975, ils représentent aujourd'hui près de la moitié de la superficie nationale totale plantée. En Thaïlande, environ $76 \%$ de la superficie totale de la palmeraie mature était entre les mains des petites exploitations en 2009 (Teoh, 2010). Les grands projets de développement en cours et à venir sont souvent basés sur des associations petits planteurs/agroindustries (ex : Sime Darby Outgrowers' Program au Libéria).

Les extensions de plantations génèrent, comme pour toutes les autres cultures de rente, des conflits fonciers, exacerbés par l'absence de relevés cadastraux ou de loi foncière précise. Les schémas de certification en cours, comme RSPO, sont conçus pour éviter ces conflits; ils offrent des recours et des bases de négociations, mais leur efficacité dépend encore fortement de l'implication des États.

\section{Planifier le développement}

Quand il est correctement planifié par les gouvernements locaux ou régionaux, le développement du palmier à huile se traduit par un fort développement économique des régions concernées et une importante réduction de la pauvreté rurale. Mal gérée, I'extension des plantations risque de se traduire par la disparition de forêts à grande valeur de conservation, avec des impacts négatifs sur les populations locales.

La mise en place de nouveaux projets de plantation, notamment en Afrique, offre aux gouvernements et à l'ensemble des parties prenantes une opportunité unique et exemplaire de développer une stratégie partagée, capable d'orienter l'expansion rapide et le développement durable du secteur. L'ensemble des acteurs (gouvernement, entreprises, centres de recherche agronomiques nationaux, communautés locales, ONG nationales et internationales) doivent élaborer une stratégie préventive.

Cette concertation doit prendre appui sur les standards élaborés par I'IFC et RSPO, qui constituent une base de travail reconnue internationalement. Diverses normes internationales sont disponibles pour aider dans cette démarche, pour la gouvernance et le contrôle-qualité (ISO 9000), le respect de I'environnement (ISO 14000) ou la responsabilité sociale (ISO 26000).

Désormais, toute stratégie d'expansion durable du secteur devra intégrer :

- I'intensification écologique des plantations existantes : diffusion de matériel végétal sélectionné, fertilisation raisonnée, bonnes pratiques agricoles ;

- la conservation de la biodiversité et du domaine forestier permanent, en privilégiant le développement de zones déjà déforestées ou dégradées ;

- I'application contrôlée des principes et critères RSPO qui doivent être interprétés en fonction des contraintes locales et intégrés dans les politiques et réglementations nationales;

- la participation des petits planteurs au développement des complexes agroindustriels, soit par la mise en place de contrats de production, soit par des mesures de soutien à l'agriculture familiale (fourniture de plants sélectionnés et de fertilisants, microcrédit, encadrement technique, formation, etc.) ;

- le respect des droits des peuples autochtones et des communautés locales, en recueillant leur consentement libre et préalable, et une large communication de tout développement de nouvelles plantations;

- I'examen du droit foncier, du cadastre quand il existe et le respect de la réglementation relative à l'acquisition des terres.

\section{L'initiative RSPO (Roundtable on Sustainable Palm Oil)}

RSPO, initiative internationale multiacteurs pour la certification et la promotion d'une huile de palme durable, était à sa création en 2004, une initiative "business to business", forte d'une dizaine de membres, acteurs privés de la filière (dont Unilever) et ONG (dont WWF). En novembre 2005, les 8 principes et 39 critères de certification ont été approuvés, conduisant à la certification des premières plantations dès 2008. Les premiers lots de CSPO (RSPO Certified Sustainable Palm Oil) ont été commercialisés à la fin 2008.

La Table Ronde rassemble aujourd'hui plus de 1000 membres, répartis en sept catégories: producteurs, transformateurs et négociants, industrie, banques et investisseurs, détaillants, ONG environnementalistes, ONG sociales.

Des groupes de travail permettent de faire vivre RSPO, de poursuivre, diversifier et améliorer ses activités, d'intégrer ses principes et critères dans les systèmes juridiques nationaux et enfin de les adapter aux contraintes spécifiques des exploitants familiaux.

Aujourd'hui, 1,5 Mha de plantations sont certifiés RSPO, soit $10 \%$ environ de la surface mondiale plantée (contre 106000 ha en 2008) et plus de 7,2 Mt d'huile de palme CSPO ont été produites en 2012 (contre 620000 t en 2008).

Les principes et critères RSPO :

- Principe 1 : Engagement de transparence

- Principe 2: Respect des lois et règlements en vigueur

- Principe 3 : Engagement du maintien de la viabilité économique et financière à long terme

- Principe 4 : Engagement des planteurs et usiniers à suivre les bonnes pratiques

- Principe 5 : Responsabilité environnementale et conservation des ressources naturelles et de la biodiversité

- Principe 6 : Gestion responsable des employés et des individus et communautés affectés par les plantations et les usines

- Principe 7 : Développement responsable de nouvelles plantations

- Principe 8: Engagement a une amélioration continue dans les secteurs d'activité majeurs

\section{L'approvisionnement en huile de palme durable}

Les transformateurs disposent aujourd'hui de quatre stratégies différentes 


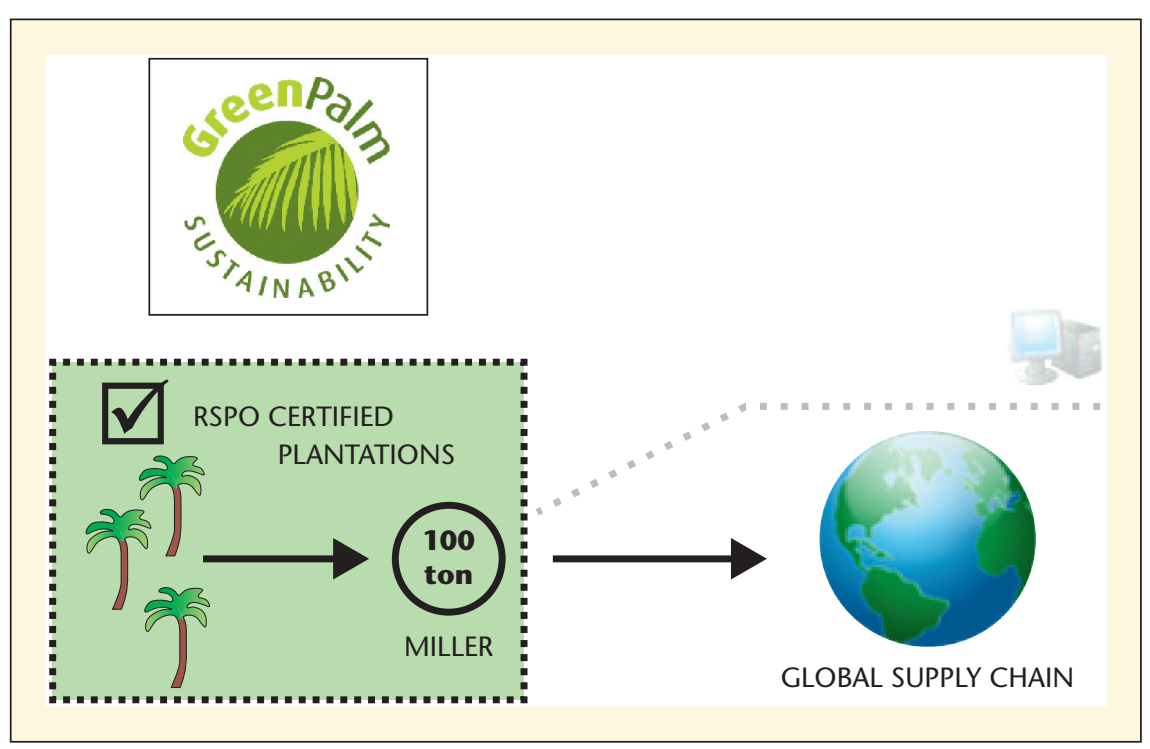

Figure 6. Les certificats Greenpalm n'attestent pas que le produit contient des matieres premieres produites durablement, mais que leur production a soutenu la production durable.

d'approvisionnement en huile durable certifiée par RSPO (CSPO : Certified Sustainable Palm Oil), présentant des degrés de rigueur et des coûts d'implémentation différents.

Pour le consommateur final, leur affichage n'est pas neutre et peut entretenir une certaine confusion: dans certains cas, le produit acheté peut contenir qu'une part limitée d'huile de palme certifiée durable. Ceci est le cas pour d'autres schémas de certification, comme le montre l'étiquetage FSC (Forest Stewardship Council) qui ne garantit pour ses Sources Mixtes qu'un "groupe de produits issus de forêts bien gérées et d'autres sources contrôlées ", un concept bien vague, même pour le consommateur attentif.. .

\section{Commande et Demande (Book and Claim)}

Les fabricants et détaillants incorporant de I'huile de palme dans leurs produits font une offre en ligne pour acheter des certificats Greenpalm et versent (via RSPO) une prime directement aux producteurs, destinée à encourager la production durable et à financer la certification de nouveaux membres (Norman, 2010). Même si le produit final n'est pas vendu comme " durable " sur le marché, les producteurs reçoivent une prime pour l'adoption de pratiques durables. Les certificats Greenpalm n'attestent pas que le produit contient des matières

premières produites durablement,
Etiquetage : "Contribue à la production d'huile de palme durable certifiée, www.rspo.org " (figure 6).

\section{Bilan de masse (Mass Balance)}

Contrôlée par UTZ Certified, cette certification correspond à l'emploi d'un mélange d'huile de palme durable et non-durable à tout stade de la chaîne d'approvisionnement, pourvu que les quantités globales au niveau de la compagnie soient contrôlées. Le modèle est construit de telle manière que les volumes de CSPO quittant la plantation ne dépassent jamais les volumes reçus par I'utilisateur final. Un contrôle indépendant des achats et ventes de CSPO et ses dérivés est effectué de manière indépendante. Aucune exigence de stockage distinct ou de contrôles dans le processus de production n'est exercée.

Etiquetage : «Mixte-Contribue à la production d'huile de palme durable certifiée, www.rspo.org " (figure 7). mais que leur production a soutenu la production durable. Ces certificats montrent l'engagement des détaillants dans la production durable au début de chaine de production, et ces derniers peuvent le faire valoir sur leur déclaration de responsabilité sociale, sur leurs emballages, dans leurs points de vente ou sur leur site internet.

\section{Ségrégation (Seggregated)}

Ce modèle, agréé par UTZ Certified, assure que I'huile de palme CSPO et ses dérivés livrés à l'utilisateur final proviennent uniquement de sources certifiées RSPO. Il autorise le mélange d'huile CSPO provenant de plusieurs sources, si elles sont toutes certifiées.

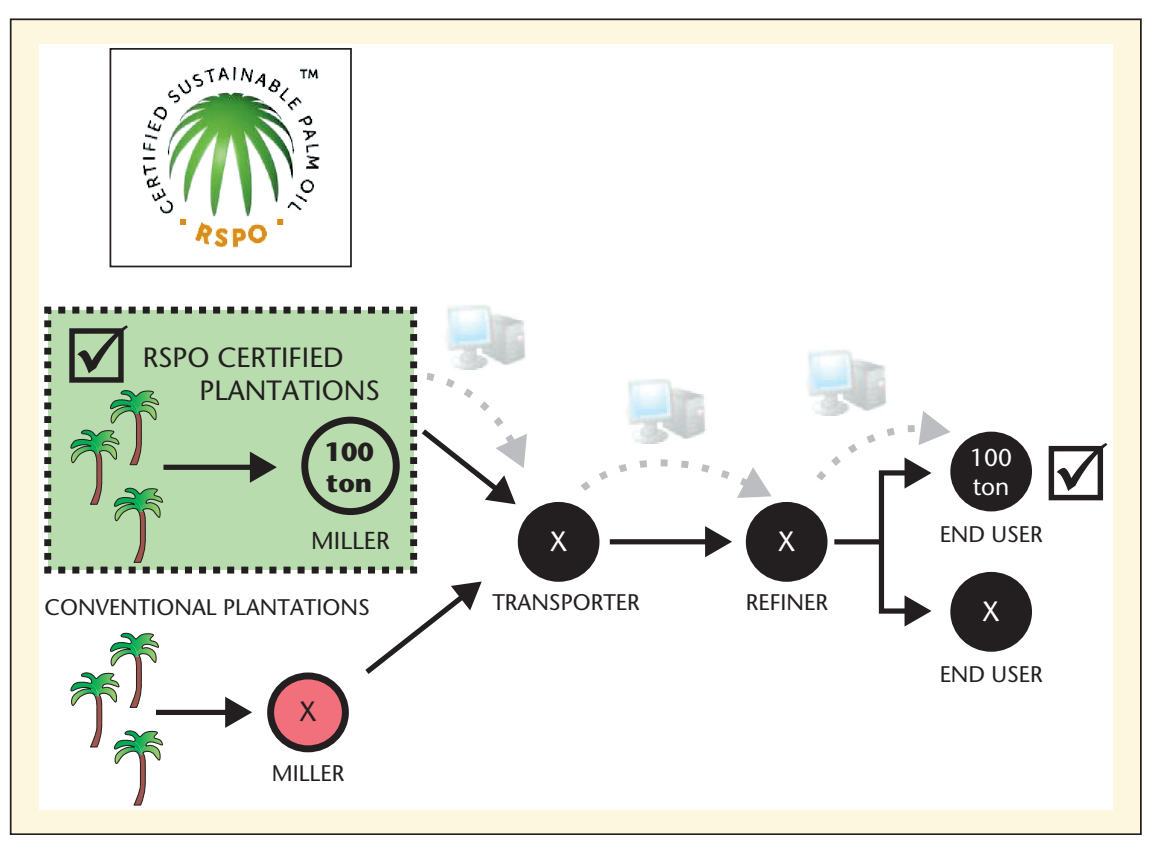

Figure 7. La certification "Bilan de masse" (Mass Balance) correspond à l'emploi d'un mélange d'huile de palme durable et non-durable à tout stade de la chaîne d'approvisionnement, pourvu que les quantités globales au niveau de la compagnie soient contrôlées. 


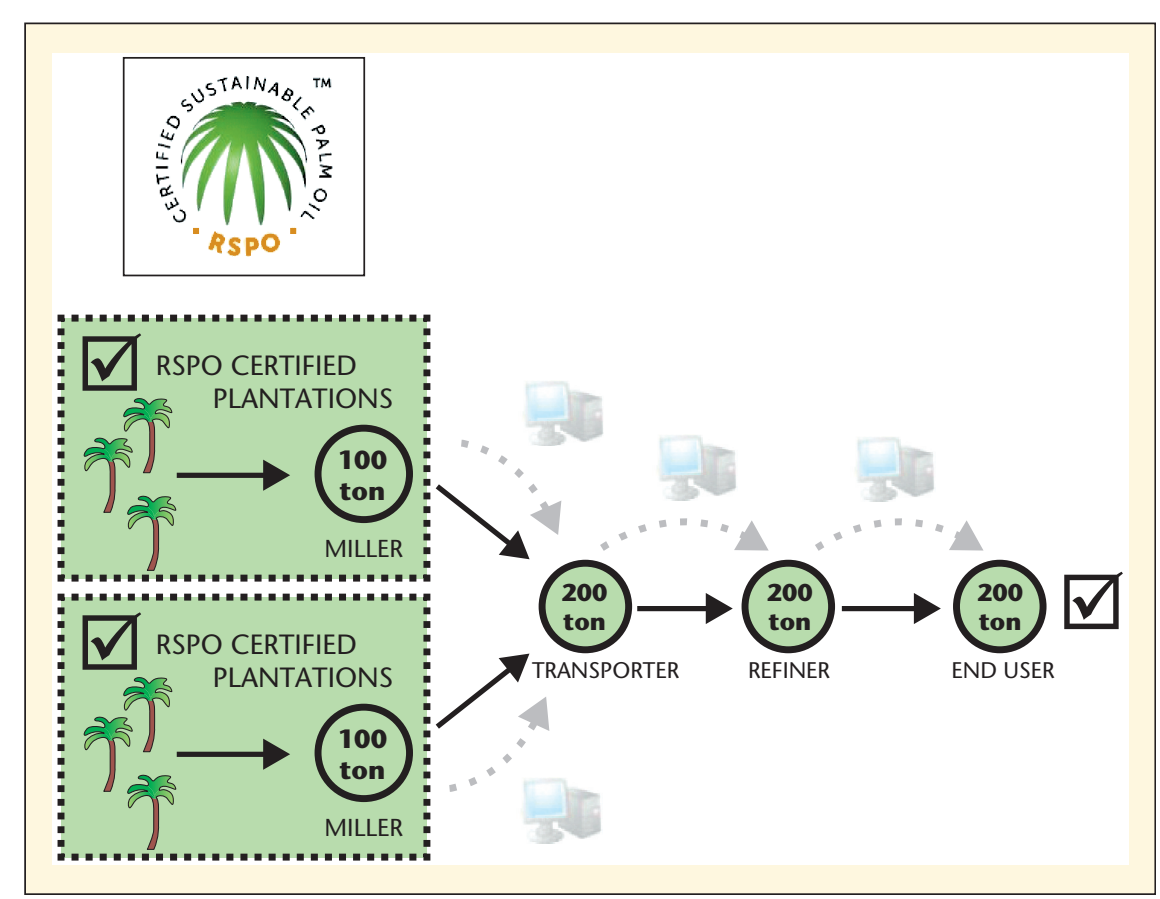

Figure 8. Ce certificat dit "ségrégation" (Seggregated) assure que I'huile de palme CSPO et ses dérivés livrés à I'utilisateur final proviennent uniquement de sources certifiées RSPO.

Ce type de chaîne d'approvisionnement en ségrégation garantit que $100 \%$ du produit physique provient de plantations et d'usines certifiées. Cependant, I'huile physique ne sera pas être pas issue d'une source unique, comme dans le cas du modèle IP.

Etiquetage : "Certifié-Contient de I'huile de palme certifiée RSPO, www. rspo.org » (figure 8).

\section{Identité préservée (Identity Preserved)}

Le modèle d'approvisionnement IP assure que I'huile de palme CSPO et ses dérivés livrés à I'utilisateur final sont issus d'une huilerie et $d^{\prime}$ 'une base $d^{\prime}$ approvisionnement unique et identifiable, et qu'ils restent physiquement isolés d'autres sources d'huile de palme tout au long de la chaîne d'approvisionnement (y compris d'autres sources de CSPO). II requiert que le producteur, le transporteur, le raffineur, et la chaîne $\mathrm{d}^{\prime}$ approvisionnement maintiennent la séparation complète et totale et la traçabilité, du lieu de production jusqu'à I'utilisateur final. Ce dernier est assuré que $100 \%$ de I'huile de palme physiquement reçue émane d'une source unique, identifiable, et certifiée RSPO. En effet, la prime actuelle (0,6 USD par
Etiquetage : "Certifié-Contient de I'huile de palme certifiée RSPO, www. Les bénéfices indirects de la certification RSPO viennent d'être analysés (WWF, 2012) : cette étude montre clairement les bénéfices, au-delà du simple bonus sur le prix d'achat de l'huile certifiée, de tonne d'huile) est largement insuffisante pour couvrir les coûts de certification, mais les bénéfices indirects sont très importants en termes d'organisation de la compagnie ou de la coopérative, de normalisation des procédures, de sécurité au travail, etc. Pour les coopératives associant les petits planteurs, les gains en productivité suite à la certification sont importants (WWF, 2012a).

Malgré ses faiblesses avérées (Laurence et al., 2010 ; Angerand, 2011), et qui sont communes à nombre d'initiatives privées multi-acteurs même plus anciennes, RSPO a toutefois l'immense mérite de jeter les bases d'un dialogue constructif à l'intérieur de la filière. Elle propose des outils imparfaits mais utiles à la moralisation de chaîne de production et son évolution vers plus de durabilité. Ces outils de certification sont encore largement qualitatifs et devront, pour gagner en crédibilité, s'affiner et se consolider sur la base de résultats scientifiques solides, partagés et reconnus. Une bonne partie des recherches collaboratives en cours sur le palmier à huile s'attachent à l'établissement de ces indicateurs robustes de durabilité.

La Table Ronde RSPO vient de se lancer dans une révision en profondeur de ses Principes et Critères, qu'il est nécessaire d'adapter aux contraintes spécifiques aux petites exploitations. Il est nécessaire de mieux associer des parties prenantes encore peu représentées: gouvernements, importateurs et transformateurs

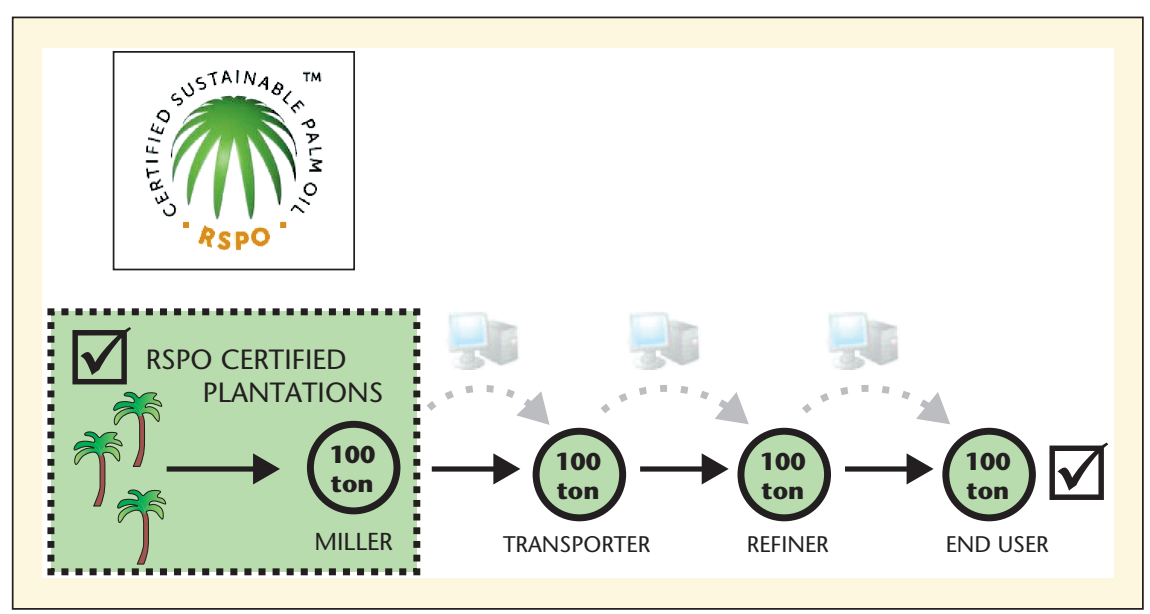

Figure 9. Le certificat "Identité préservée" (Identity Preserved) assure que I'huile de palme CSPO et ses dérivés livrés à l'utilisateur final sont issus d'une huilerie et d'une base d'approvisionnement unique et identifiable, et qu'ils restent physiquement isolés d'autres sources $d$ 'huile de palme tout au long de la chaîne d'approvisionnement (y compris d'autres sources de (SPO). 
du Sud (Chine, Inde, Pakistan), petits planteurs, universités et centres de recherche nationaux et internationaux. . .

RSPO devra veiller à donner une part importante aux gouvernements, car ce sont bien eux qui, in fine, sont en charge de légiférer et faire appliquer les lois encadrant les critères de durabilité dans chaque pays (moratoires sur l'exploitation des forêts, standards obligatoires, concessions foncières).

L'émergence récente de standards nationaux obligatoires (ISPO : Indonesian Sustainable Palm Oil; MSPO : Malaysian Sustainable Palm Oi) est le signe d'une appropriation réussie de la certification, mais aussi de la nécessité de se doter de règles nationales obligatoires et non plus volontaristes. À la différence de la certification RSPO, ces standards nationaux répondent à une initiative gouvernementale et non privée. Ils correspondent à une compilation des textes de lois régissant la filière, leurs conditions d'application et les sanctions aux contrevenants. À l'échelle nationale, leur caractère légal et obligatoire leur donne une plus large audience que la certification volontariste RSPO. Ce cadre rigide ne permet toutefois pas la souplesse nécessaire à une adaptation rapide aux évolutions de la filière. Certaines ONGs ont critiqué ces initiatives nationales, car si elles apparaissent comme rigoureuses et détaillées sur l'encadrement des bonnes pratiques agricoles, elles le sont moins en termes de conservation des espaces protégés et de protection des droits des populations autochtones (Gillespie et Harjanthi, 2012).

Les investisseurs ont également un rôle clef à jouer dans le développement durable de la filière, en conditionnant leur soutien à la prise en compte des questions de gouvernance et de respect des normes sociales et environnementales et à la certification RSPO de leurs bénéficiaires (WWF, 2012b).

\section{Les recherches en cours}

L'intensification écologique de I'exploitation d'une plante pérenne telle que le palmier à huile requiert des investissements dans la recherche sur le très long terme. Ainsi, I'amélioration génétique du palmier à huile est un processus récurrent, engagé depuis plus de 50 ans et mobilisant des milliers $d^{\prime}$ 'hectares d'essais génétiques dans le monde, dont les résultats sont exploités en réseau. Les recherches en génétique font désormais appel aux biotechnologies (micropropagation, sélection assistée par marqueurs, recherche de gènes, cartographie du génome) afin d'accélérer la création du progrès génétique et sa diffusion auprès des utilisateurs finaux.

L'amélioration variétale progresse sans faire appel aux OGM : la transformation génétique est particulièrement délicate chez les monocotylédones comme les palmiers. Même si la faisabilité technique de cette transformation a été démontrée en Malaisie il y a près de 20 ans, il n'existe pas de trait simple à améliorer de cette manière. En effet, vitesse de croissante, rendement en huiles ou qualité des huiles sont des caractères multigéniques et plus ou moins bien héritables; or pour générer des OGM utiles, il faut des caractères gouvernés par une ou une poignée de gènes seulement. En outre, I'huile de palme est en passe de devenir la seule huile végétale majeure non-OGM sur le marché, un argument commercial qui pèse son poids...

Dans un contexte de changements climatiques en cours ou à anticiper, les producteurs souhaitent se doter d'un outil fiable de prévision du rendement (Combres et al., 2012). Les travaux de modélisation de l'impact des changements climatiques globaux (dont les épisodes El Niño/La Niña) sur le rendement des plantations demandent des séries de données météorologiques et des calculs de rendements dans plusieurs environnements agroécologiques diversifiés et sur de très longues séquences (plusieurs décennies). Ces recherches sur le très long terme reposent sur des réseaux et des partenariats bilatéraux Nord/Sud et publics/privés très actifs.

Les critères d'évaluation et de certification des plantations de palmier à huile doivent être robustes et acceptés par l'ensemble des parties prenantes. Ils doivent donc reposer sur des indicateurs agri-environnementaux simples reposant sur bases scientifiques solides (Caliman, 2011). Les principes et critères de la certification font trop peu souvent appel à des données chiffrées : il existe donc un besoin de connaissance à combler par des études multidisciplinaire de grande ampleur. Au cours de la dernière décennie, plusieurs projets collaboratifs d'envergure ont été lancés afin de déchiffrer les bases biologiques, agroécologiques et sociales de la durabilité de l'élaeiculture.

Ainsi, le projet SEnSOR (Socially and Environmentally Sustainable Oil palm Research) coordonné en Asie du SudEst par la Royal Society of London, développe une approche multidisciplinaire pour pallier au manque de connaissances scientifiques dans la validation et le développement des Principes et Critères RSPO. Le projet rassemble les universités européennes de York, Wageningen, Leeds, Lancaster et Swansea et s'adresse a cinq sujets majeurs : Eaux et sols, Qualité de l'air et gaz à effet de serre, Biodiversité, Zones à Haute Valeur de Conservation, Droits et Procédés participatifs et Niveau de vie. Ses objectifs principaux sont de : 1) Générer de la confiance envers I'huile de palme certifiée RSPO chez les utilisateurs, les investisseurs et le grand public, 2) Garantir que les efforts et les investissements dans des pratiques durables consentis par les planteurs sont rentables et reconnues sur le marché et 3) Protéger l'environnement et la société sur le long terme, grâce à l'évaluation rigoureuse et au développement de pratiques capables de donner des résultats prouvés.

Sur l'île de Bornéo, le projet SAFE (Stability of Altered Forest Environment) est mené sur 10 ans (2010-2020) par la Royal Society of London et la Fondation Sime Darby, pour un budget de 7,3 millions d'euros. II s'agit d'un projet de recherche en écologie de très grande envergure, destiné à quantifier les impacts de l'installation de plantations de palmier à huile sur la biodiversité des forets adjacentes. II s'agit d'identifier les situations permettant de minimiser les impacts sur la biodiversité et d'optimiser les services écosystémiques. Les programmes de recherches s'intéressent à la diversité de la flore et de la faune, l'eau et le sol, le cycle du carbone et des nutriments et le microclimat, sur des blocs forestiers de 1, 10 et 100 hectares préservés à l'intérieur d'une plantation de palmier à huile. Les zones d'études seront installées dans de nouvelles plantations, des plantations en production et des zones déforestées et des parcelles de forêt primaire.

Afin de générer des indicateurs partagés sur les impacts sociaux et environnementaux de la culture du palmier à huile, le Cirad et ses partenaires (INRA, Cenipalma, PT Smart, NBPOL) ont créé le 
Réseau PalmiNet. Cette initiative est destinée à promouvoir l'usage des indicateurs auprès des acteurs de la filière, à mettre en commun les efforts de recherche et les retours d'expérience, à faciliter le développement d'outils et d'indicateurs, à faciliter l'émergence de nouvelles collaborations et de recherches multidisciplinaires, et à diffuser ses résultats auprès d'un large public d'utilisateurs potentiels.

À l'interface entre agronomie et sciences sociales, le Projet SPOP (Sustainable Palm Oil Production), financé par l'Agence Nationale de la Recherche, vise à mettre en commun des compétences (INRA, IRD, Cifor, Cirad) afin de : 1) fournir de nouvelles connaissances scientifiques et des outils pour évaluer les impacts économiques, environnementaux et humains des différents systèmes de culture du palmier à huile pour vérifier la durabilité des systèmes actuels ou proposer de nouveaux systèmes durables; 2) impliquer les acteurs dans le processus à l'aide de méthodes participatives telles que la modélisation multi-agent ; 3) d'identifier les obstacles et d'analyser leur mécanismes, à savoir s'ils sont liés à l'incapacité intrinsèque de certains systèmes à s'adapter ou à l'échec ou les difficultés liés à la mise à disposition des connaissances et outils pour les acteurs.

L'interprétation locale et I'application des Principes et Critères RSPO soulèvent ainsi de nombreuses questions à la recherche. À terme, c'est l'impact de la certification elle-même qui devra être quantifié et interprété en utilisant des indicateurs précis.

\section{Quel rôle pour les consommateurs du Nord?}

Les consommateurs occidentaux ne sont en contact qu'avec la partie ultime de la chaine de transformation, mais aussi la plus sensible. Ils peuvent tirer la filière vers le haut, en exigeant des transformateurs le respect des normes existantes de durabilité, même si elles sont perfectibles. II importe de privilégier et d'encourager l'utilisation d'huile certifiée RSPO (et/ou soumise à d'autres standards plus drastiques, si disponibles et vérifiables). Sous la pression des consommateurs, de nombreuses enseignes et industriels européens se sont engagés à utiliser $100 \%$ d'huile certifiée d'ici 2015, c'est un progrès très important. Toutefois, la moitié seulement de l'huile de palme certifiée mise sur le marché est effectivement achetée, il faut donc accélérer cette tendance pour encourager producteurs et distributeurs à entrer dans une coûteuse démarche de certification.

Les stocks sont très faibles $(<8 \%$ de la production en 2012) et la demande est soutenue par des pays a forte croissance économique et démographique. Retirer du marché les $17 \%$ destinés aux besoins du Nord (si on inclut les biocarburants) aura pour effet de regonfler l'offre en la débarrassant de toute contrainte de certification, absente des marchés du Sud. L'effet sera l'inverse de celui escompté : on encouragera alors indirectement la production $\mathrm{d}^{\prime}$ huile de palme non certifiée durable.

Boycotter I'huile de palme, c'est aussi encourager la production d'autres huiles végétales de composition chimique différente, souvent OGM, et pas forcément plus écologiquement ou socialement acceptables.

\section{Conflits d'intérêts : aucun}

\section{RÉFÉRENCES}

Angerand $S$. Arnaque à I'huile de palme durable : 12 questions pour comprendre les enjeux. 2011. http://www.amisdelaterre.org/ IMG/pdf/rapport_arnaque_huile_de_palme_ durable_mai_2011.pdf

Caliman JP. Palmier à huile : le management environnemental des plantations. OCL 2011 ; $18: 123-31$.

Carlson KM, et al. Committed carbon emissions, deforestation, and community land conversion from oil palm plantation expansion in West Kalimantan, Indonesia. PNAS 2012 ; 109 : 7559-64 doi: 10.1073/ pnas. 1200452109.

Combres JC, Pallas B, Rouan L, et al. Simulation of inflorescence dynamics in oil palm and estimation of environment-sensitive phenological phases: a model based analysis. Functional Plant Biology 2012 ; 40 : 263-79.

Corley RHV. How much palm oil do we need? Environmental Science \& Policy $2009 ; 12$ : 134-9.
Durand-Gasselin T, Cochard B, Amblard P, De Franqueville $H$. Un regard sur quarante ans d'amélioration génétique du palmier à huile (Elaeis guineensis) et son impact sur la filière. Le sélectionneur français 2002 ; 53 : 133-47.

FAO. 2010. Global Forest Resources Assessment 2010: Main Report. (Food and Agric Org of the UN, Rome, Italy).

Gillespie P, Harjanthi RS. ISPO, RSPO: Two sides of the same coin? The Jakarta Post 2012 ; November $02: 6$.

Hoyle D, Levang P. 2012. Le développement du palmier à huile au Cameroun. 16 p. http:// awsassets.panda.org/downloads/developpmentpalmierhuilecameroun.pdf.

Jacquemard JC. Le palmier à huile. Agricultures tropicales en poche. CTA, Presses Agronomiques de Gembloux, 2012

Koh LP, Levang P, Gazhoul J. Designer landscapes for sustainable biofuels. Trends in Ecology and Evolution 2009 ; 24 : 431-8.

Laurence WF, et al. Improving the Performance of the Roundtable on Sustainable Palm Oil for Nature Conservation. Conservation Biology 2010 ; 24 : 1523-739http://dx.doi. org/10.1111/j.1523-1739.2010.01448.x

Norman B. Greenpalm: Une approche innovante pour l'huile de palme durable. 2010. http://www.greenpalm.org/upload/ files/18/GreenPalm_4pp_French.pdf.

Rival A, Jaligot E. Oil palm biotechnologies are definitely out of infancy. OCL $2010 ; 17$ : 368-74.

Sayer J, Ghazoul J, Nelson P, Boedhihartono AK. Oil palm expansion transforms tropical landscapes and livelihoods. Global Food Security 2012.

Teoh CH. Les Principaux Enjeux du Développement Durable dans le Secteur de I'Huile de Palme. International Finance Corporation - World Bank Group, 2010, 53 p. http://www.ifc.org/ifcext/agriconsultation. nsf/AttachmentsByTitle/Discussion+Paper French/\$FILE/Discussion+Paper_French_ FINAL.pdf

WWF. Huile de palme : de la déforestation à la nécessaire durabilité. Rapport, 2011, 40 p. http://wwf.fr/media/files/rapport-huile-depalme-2011.

WWF. Sustainability in Palm Oil Production: Analysis of Incremental Financial Costs and Benefits of RSPO Compliance. 2012a. http:// assets.worldwildlife.org/publications/350/ files/original/Profitability_and_Sustainability_ in_Palm_Oil_Production.pdf?1345734683

WWF. 2012b. http://awsassets.panda.org/ downloads/wwf_palmoil_investorreview.pdf 
Annexe 1

Sites web utiles :

- RSPO - Roundtable on Sustainable Palm Oil: www.rspo.org/

- Round Table on Responsible Soy Association: www.responsiblesoy.org/

- FSC Forest Stewardship Council: www.fsc.org/

- Dossier Palmier à huile et Développement Durable: Oléagineux, Corps Gras, Lipides: www.revue-ocl.fr/archives/ sommaire.phtml?cle_parution $=\mathbf{3 4 4 7}$

- Stability of Altered Forest Ecosystems (SAFE) Project: www.safeproject.net/

- Palm Indicators Network - PalmiNet :

http://community.plantnet-project.org/pg/groups/2879/palminet/

- Greenpalm: http://www.greenpalm.org/

- Sensor: http://www.searrp.org/sensor/

- SPOP : Sustainable Development of Palm Oil Production: Designing strategies from improved knowledge on oil palm cropping systems

- http://www.agence-nationale-recherche.fr/programmes-de-recherche/environnement-et-ressources-biologiques/viabilite-et-adaptation-des-ecosystemes-productifs-territoires-et-ressources-aux-changements-globaux/fiche-projet-agrobiosphere/?tx_Iwmsuivibilan_pi2\%5BCODE\%5D=ANR-11-AGRO-0007 